\title{
PSYCHOLOGY OF SAFETY AND RESISTANCE TO TERRORISM
}

Aleksander Yu. Kipiani Mikhail V. Potapov

Lomonosov Moscow State University Moscow

\begin{abstract}
In the article main parameters of any emergency situation that influence the psychological stress intensity are described. The most frequent consequences of emergency situations are discussed. The characteristic of psychological and psychosomatic reactions of the rescuers and secret service agents is provided. Professional and personal training of emergency service workers involved in work with victims of terrorist attacks and emergency situations is described. Attention is drawn to the the problem of interaction between emergency service workers and victims.
\end{abstract}

Key words: emergency situations, emotional burnout syndrome, motional crisis, negative effects, psychology of Safety, psychological stress, psychological protection, terrorism

\section{Psychographics of survivors of emergency situations}

Besides injuries, emergency situations cause psychological stress.

Below are listed the main parameters of any emergency situation that influence the psychological stress intensity: 1 ) hostility of the situation (danger to life, physical and mental integrity of the individual or that of his/her relatives); 2) suddenness; 3) intensity of negative effects; 4) long duration of negative effects; 5) ambiguity and unpredictability of the situation; 6) impossibility of taking control of the situation (the situation develops against the individual's will); 7) lack of social support; 8) aversion to his/her own behavior in an emergency situation; 9) incompatibility of the new experience with habitual reality; 10) impossibility of explaining the situation rationally. 
Let us characterize the most frequent consequences of emergency situations.

1. Changes in the affective sphere. Disturbances of the emotional sphere may manifest themselves in emotional "dullness", depression, dismal mood, and inability of experiencing joy, love, and creative enthusiasm. One constantly feels helpless and doesn't know what $\mathrm{s} / \mathrm{he}$ is supposed to do. Intrapersonal conflicts and crises become more intense. The feeling of shame and guilt arises; the effect of survivor's guilt is widespread among survivors: the person blames himself/herself for surviving the traumatic situation that cost others their lives.

2. Changes in the cognitive sphere. The survivor's memory deteriorates, $\mathrm{s} /$ he becomes amnesiac and cannot recall the traumatic events and experiences problems with concentration.

3. Changes in behavior. The person's behavior becomes illogical, inconsistent and unpredictable. Excessive activeness and hypervigilance (detecting threats becomes the person's dominant trait) may be observed. Innocuous but sudden stimuli may produce a reaction of fear: the person starts behaving in a way as if $\mathrm{s} / \mathrm{he}$ were in danger.

\section{Psychological reactions of specialists, who deal with the consequences of terrorist attacks, as the result of work with victims of emergency situations}

The first people who get in contact with the victims of accidents and terrorist attacks are rescuers and secret services agents.

Meeting a person with a traumatic experience always produces strong personal reactions. Taking account of such reactions is an indispensable condition for an effective professional activity of those who come to the rescue of survivors. It is also an essential parameter of protecting doctors and their patients.

In the second half of the XXth century psychologists started examining the reactions of helpers (rescuers in the first place) to mass traumas and deaths.

The following characteristic psychological and pshycosomatic reactions of the rescuers and secret service agents were described:

1. Irritability: the person feels weak and can't do anything; the effectiveness of his/her activity decreases; every now and again causeless grudge against somebody or something arises. 
2. Tachycardia: the person knows that s/he is in very good health but feels pain in the chest and symptoms of heart attack.

3. Nervous tremor.

4. Inability to act rationally: the person suddenly loses the ability to exercise his/her duty normally and can't remember his/her official responsibilities.

5. Bursting into tears.

6. Anxiety: the person takes up different activities and cannot differentiate important from unimportant.

7. Exhaustion: suddenly, the person feels devastated, cannot take a single step, and wants to sit down. Every single muscle aches.

8. Pestering: the person feels the need to talk and to share his/her terrible experience with somebody. S/he tends to retell the same story over and over again.

9. Escape reaction.

10. Despair: the person loses his/her self-control, breaks down, feels dizzy, and wants to hide in a secluded place.

Specialists use different methods to prevent and neutralize the negative psychological consequences of work with victims of emergency situations. One of the most spread methods is debriefing. Participation in constant rescue operations may cause deformations in one's professional activity and negative changes in one's mental health. In the modern literature these negative consequences are commonly referred to as emotional burnout.

The burnout phenomenon. Those whose duty is to help people in emergency situations, and especially those who constantly deal with other people's grief and sufferings, sooner or later experience psychological problems that influence their emotional state and professional behavior. This is the so-called emotional burnout syndrome.

The emotional burnout syndrome has three components:

1. The feeling of emotional exhaustion. As emotional exhaustion accumulates, emergency service workers feel that they can't devote themselves to people as much as before.

2. The development of negative attitude towards victims. This starts with negative remarks about victims in conversations with colleagues. Then, emergency service workers begin feeling a strong dislike for victims, which they try to repress, but don't really succeed. That results in 
fits of anger and open hostility. Maslach refers to this process as "dehumanization" or "depersonalization".

3. The development of negative attitude towards the job and self. Maslach calls this component "the reduced sense of personal accomplishment"

\section{Professional and personal training \\ of emergency service workers involved in work with victims of terrorist attacks and emergency situations}

Providing help to people in emergency situations is a high risk and high stress job. On the one hand, victims of terrorist attacks and other emergency situations require special treatment and care as well as undivided attention. On the other hand, emergency service workers who work with them may be subjected to health risks, both physical and mental. In this connection, a complex psychological approach to emergency service workers is indispensable. Such an approach should be directed to: firstly, the development of high tolerance as the way of interaction with victims; and secondly, the prevention of possible health risks.

The problem of interaction between emergency service workers and victims has two important aspects: 1) the development of personality traits characterizing a tolerant person in an emergency service worker 2) the formation of tolerant attitude towards patients.

General approach to the patient: a victim should be regarded as "a subject", but not as "an object". A formed attitude towards victims is a key factor.

Communication with the patient: one of the most important aspects of the "doctor-patient" relations is the ability to talk to the patient correctly.

To a great extent, the effectiveness of work with a patient relies on the patient's confidence level, which is, for the most part, determined by the doctor's ability to communicate correctly with him/her. Important information about the patient may be collected through the following: superfluous information given by the patient to the personnel, introductory commentaries and physical appearance: clothes, hair style, gesticulation, mimicry, intonation, facial expression, talkativeness, irritability, hostility, aggressiveness, depression, need for being accompanied by relatives. 


\section{Coping with the negative psychological consequences of long contact with victims of emergency situations}

At some stage, any practitioner has to develop psychological protection against being too emotional about the sufferings of patients. After taking emotionally and to heart patients' sufferings, deaths, operations, bleedings and mental disorders, etc. at the beginning of working life, doctors, nurses and support personnel acquire some emotional resistibility with time. Although a certain proportion of emotional resistibility is necessary and vital, a specialist should retain the ability to perceive a patient as a person who is suffering and have respect for the dead. There is a danger of the development of professional psychological deformations: in particular, treating patients with indulgent disdain, aggression and ignoring their feelings.

To cope with the emotional crisis, emergency service workers may apply the same methods as they do when treating patients. W. Kirk suggests using the following "surviving tool kit":

1. Accept what happened.

To accept the reality of what happened, three strategies may be applied:

a. Don't isolate yourself from others, thinking that there is something wrong with you as your reactions seem to be improper;

b. Share your feelings with someone from your family, who will to listen to you and accept your tears, anger and apathy. Tell him/her about the negative experience you went through.

c. Discuss your feelings with your colleagues. They are likely to have similar feelings. You will help each other to put up with the reality.

2. Accept your reactions and feelings.

Some of your feelings and reactions may be completely new to you, but they are familiar to those who have gone through the same crisis. Even the feelings that you regard as strange and incongruous or "wrong" and "evil" are quite natural in the situation you went through.

3. Learn a lesson from what happened.

The situation of crisis has opened a new life dimension to you and taught you something new. Take advantage of this knowledge in your future life and work.

4. Direct your feelings at something new. 
The first three steps will help you to release the energy that has previously been used to depress your feelings. This energy may go into other feelings. The condition of crisis may lead to sole-searching, make you life even richer and broaden your horizons.

If a specialist finds it impossible to cope with the crisis on his own, he should turn to a psychologist or a psychotherapist for help.

The training program for emergency service workers, providing help to victims of terrorist attacks, may include the following: holding debriefings, Balint groups, trainings, aimed at the development of cultural sensitivity and the doctor-patient communication skills, and trainings aimed at preventing stress and emotional burnout.

Debriefing is a group method of urgent psychological help to people, who have experienced a traumatic event. For the first time, it was introduced shortly after World War I to provide crisis therapy to victims of catastrophes. It has been widely used ever since. A debriefing is an arranged meeting for people, who experienced the same extreme and stressful event, where they discuss it (including the rescuers and medical staff involved in work with the victims). The purpose of debriefing is to minimize the psychological harm of the stressful event. Debriefing is a method of crisis intervention and prophylaxis, which, however, can't completely prevent the consequences of the stressful and traumatic experience, but can reduce them. It contributes to a better understanding of the causes and to making reasonable decisions. This method allows to solve the following problems:

- "psychological ventilation" - the analysis of feelings and emotional reactions;

- the improvement of the cognitive processing of the experience through understanding the event and personal reactions to it;

- reducing individual and group emotional strain;

- escaping from the feeling that one's emotional reactions are pathological and unique;

- mobilization of internal and external resources to cope with the consequences of stress;

- preparation for coping with long-term symptoms and reactions;

- determining the methods of further help in case of need.

It is recommended to take part in a debriefing every time after experiencing a stressful and traumatic incident. The optimal time for a debriefing varies, according to different sources. 
A debriefing may be held under the guidance of a psychologist or a person from the team of rescuers - a doctor or an officer - who has received appropriate training (and is familiar with the methods of working with a group and with the problems related to experiencing a trauma or grief). A debriefing should be held by one person or together with an assistant. Any secluded place isolated from outsiders is appropriate for holding a debriefing.

The number of participants is 10 to 15 people. The duration is 2 hours. If there are more than 15 participants, it is better to work simultaneously in small groups. Debriefings are held without a break - that is why the participants are recommended to take some food and go to the toilet before it starts.

The simplest ways to cope with stress are relaxation techniques, which should be mastered by all rescuers involved in work with victims of emergency situations.

Relaxation techniques are methods, aimed at creating conditions for the development of skills at voluntary self-regulation of the physiologi$\mathrm{cal}$ and mental functions. The best-known techniques of relaxation were elaborated in the 1920s and include autogenic training and progressive muscle relaxation.

Progressive muscle relaxation is a series of simple exercises. An exercise consists in, first, tensing a muscle group for a few seconds and then releasing it. Tensing and releasing various muscle groups throughout the body produces a deep state of physical relaxation, accompanied by a feeling of warmth and heaviness in muscles. Physical relaxation is proved to be effective against negative emotions.

Progressive muscle relaxation is capable of relieving a variety of conditions caused by stress, such as insomnia, high blood pressure, neurosis and headaches. Progressive muscle relaxation improves a person's ability to have control over mental state, enables the formation of internal control devices for the management of mental functions and creates the necessary prerequisites for mastering more complicated techniques of psychical regulation.

Autogenic training is a relaxation technique aimed at balancing homeostasis, disordered by stress. Autogenic training includes two steps, the lower and the higher. The lower step teaches you to create a feeling of warmth and heaviness throughout your body and manage your heart and breathing rate. The higher step is directed to creating states of trance 
of different levels. The fundamental mechanism of autogenic training consists in the formation of strong links between orders to the self and creation of certain states in different psychophysiological systems.

Progressive muscle relaxation requires no special training and can be practiced by any person at once. As for autogenic training, it requires previous training and serves as a more active way of affecting one's psychophysiological and emotional state.

Apart from relaxation, breathing exercises and the elementary selfmassage skills should also be mastered by emergency service workers as the effective ways of reducing stress.

Very often, a burnt out person doesn't notice his state of mental health, and the symptoms become more obvious to his colleagues. The more the syndrome progresses, the less the person wishes to recognize it. That is why it is important to reveal the problem and take appropriate measures in time. At an early stage, a simple talk with an understanding colleague may be a great help for a burnt out person, who needs help and support, but not criticism and conflicts. Burnout is not unavoidable. It can be prevented or reduced by taking well-timed measures. Many strategies that help to cope with burnout serve at the same time as its preventive measures. The preventive strategies may be divided into several levels, according to what is the focus of attention: individual workers, labour management or the organization structure as a whole.

At the level of the organization as a whole, the strategies for preventing burnout consist in a more exact and consistent formulation of the policies and goals. It is indispensable to arrange trainings for employees and research new areas. Help and care should be provided to victims in cooperation with their families, social circle (community) and partner-organizations. It is important to work out the effective mechanisms for group and organization ways of solving problems. Employees should be encouraged to work individually and to be involved in collective decisions.

The effective labour management plays a great role in preventing stress and burnout too. Hard and ungrateful work should be distributed in equal proportions among the personnel. Employees should carry out different types of work, so that one person won't be loaded with all work. The schedule of work should include a balanced alternation of easier and harder tasks, with the time for breaks, during which workers could relax and socialize. 
Debriefings, trainings, Balint groups and other meetings should be arranged to discuss ways of resolving conflicts, making collective decisions and reducing stress. In addition, trainings, aimed at self-knowledge and the development of tolerance, produce positive effect. They help the participants to understand what things in their job provoke the feelings of anger, fault or frustration in them, and to work out the adequate forms of interaction with patients.

It is highly important for the head of the organization to:

- give the personnel the opportunity for acting out the feelings that they gain when helping victims of catastrophes;

- create the system of feedback within the organization: from the lower ranks to the senior, and backwards (for instance, very important are regular meetings with the personnel where arising problems are discussed and feedback about the effectiveness of work is given);

- control the level of the personnel's emotional strain and take appropriate measures when it becomes too high.

At the level of the personnel, preventive strategies include:

- setting realistic and achievable goals so that the personnel's excessive demands to themselves can be diminished;

- providing feedback to the employees about the effectiveness of their work;

- providing trainings for the employees and opportunities to raise the level of one's skills within the organization so as to increase labour efficiency;

- teaching anti-stress techniques;

- informing new-comers about the job-related stresses;

- regular checks on the level of emotional strain to reveal the burnt out workers;

- helping employees to solve personal and job-related problems;

- creating support teams for exchanging resources as well as friendly atmosphere of support and mutual understanding within the organization.

To avoid burnout, emergency service workers must separate working and private lives. As soon as they mixed them up, working life takes a grip on and dominates all free time, and the risk burnout increases. 
Having hobbies and interests is indispensable for relaxation and filling gaps in energy.

Taking measures for preventing burnout at different levels will help emergency service workers to preserve professional qualities and skills and positive personality traits.

People, working with victims of catastrophes and refugees, often deal with people from other countries. Therefore, their training must include learning cultural sensitivity -understanding differences between cultures, ability do deal with people from other cultures, and, most importantly, tolerance towards cultural differences and releasing from stereotypes.

To develop cultural sensitivity, various techniques have been worked out. Many of them are worthy of using in trainings, like for example:

Cultural assimilator (a technique for increasing cultural sensitivity) is a technique of cross-cultural training, aimed at learning the adequate ways of interaction with people from other cultures. It is based on cognitive learning and teaching through compiled reviews. The participants of a training read short passages, describing situations (there may be from 37 to 100 situations) in which individuals from different cultures come across. These situations are recognized by experts as problematic and leading to conflicts, misunderstanding and wrong interpretations due to more or less considerable differences between the cultures. Stereotypes about the other culture, different role-playing expectations, customs and non-verbal behaviour are all taken into account when compiling the review. The situations may be taken from books on ethnography and history, the press or personal experience. After having read the situation, the participants of the training need to choose the correct interpretation of the situation out of the four suggested. Only one interpretation is recognized as correct in the view of people from the culture under investigation. If the chosen interpretation is the correct one, a participant is allowed to analyze another situation. If s/he has failed, the trainer explains the mistake and gives another chance to choose the correct interpretation. The analysis of such situations helps people to become knowledgeable about cultural standards and to understand other cultures as they are, without viewing them in the light of one's own. 